\title{
ANÁLISIS DE ENERGÍA DEL OSCILADOR ARMÓNICO CONMUTADO
}

\author{
Edgar Mendoza Parada \\ Universidad Pedagógica Nacional \\ Departamento de Tecnología. \\ edgarmen@colomsat.netco
}

\begin{abstract}
The article shows an analysis of energy variation on a variable structure system. It is composed by two harmonic oscillators of different frequencies switched when velocity is zero and velocity is maximum. In conclusion, each time less energy finite quantities are instantly lost in successive switched where velocity is y.ero, until store energy in system is voiding. In the switched where velocity is maximum, energy does not show any variation. The article also shows an analogy with a switched electrical oscillators and it shows the physical interpretation corresponding.
\end{abstract}

\section{RESUMEN}

Se presenta un análisis de la variación de energía en un sistema de estructura variable formado por dos osciladores armónicos de diferente frecuencia que se conmutan cuando la velocidad es cero y cuando la velocidad es máxima. Se concluye que cantidades finitas de energía cada vez menores son pérdidas instantáneamente en las sucesivas conmutaciones donde la velocidad es cero hasta que finalmente la energía almacenada en el sistema se anula. En las conmutaciones donde la velocidad es máxima la energía no varía. Se presenta además una analogía con un oscilador eléctrico conmutado y se muestran las correspondientes interpretaciones físicas. 


\section{Introducción}

Supóngase un oscilador masa-resorte sobre una superficie sin fricción, tal que la masa o la constante elástica cambian entre dos valores. en instantes bien predeterminados de la oscilación. Esta conmutación física es de difícil realización, además claro está, que la condición de ausencia de fricción es imposible. Sin embargo, el sistema análogo eléctrico formado por una bobina y un condensador, que conmutando con otro en paralelo modifica su valor secuencialmente en tiempos preestablecidos, permite una aproximación más fácil a una realización física del problema mecánico descrito. En cualquiera de los dos casos la conmutación no introduce en el modelo matemático ningún elemento disipador de energia. No obstante, en ambos casos las variables de estado del sistema (posición y velocidad de la masa, o corriente y voltaje en el circuito) tienden a cero, como se verá fácilmente en un análisis sobre el plano de fase. Esto indica que la energia inicial del sistema desaparece. Cómo ocurre esto, en sistemas conservativos que conmutan entre si, es el problema básico tratado en este artículo. La situación es interesante desde el punto de vista pedagógico por su naturaleza paradójica, y también porque el problema se puede inscribir en el contexto del control de estructura variable, aunque normalmente en este ámbito los problemas no se abordan desde una perspectiva de energia.

En el siguiente item se describe de manera somera el entorno de control en el que el problema se formula. Después se aborda desde una perspectiva esencialmente fisica tomando como base de interpretación el oscilador masa resorte. Finalmente se analiza el oscilador bobina condensador conmutado por su riqueza explicativa. ${ }^{1}$

1. En términos matemáticos, el problema a resolver en un sistema de control puede formularse de manera bastante general, como el de la

1. No es esencial la formulación del problema de control de los siguientes párrafos para seguir la argumentación a partir de la ecuación (1) abajo. búsqueda de una función que altere de manera conveniente el campo vectorial asociado a las ecuaciones diferenciales que describen el comportamiento del sistema físico a ser controlado. Si la función de control se determina con base en el conocimiento de las variables de estado del sistema, y depende de ellas, se tiene el llamado control por realimentación de estado. En este caso la dinámica de cada variable de estado $x_{i}$, es indicada por cada una de las lineas de la ecuación $\dot{x}=f(x, t, u(x))$ con $x \in \Re^{n}$ y $u(x) \in \Re^{m}$. Si el campo vectorial no depende explicitamente del tiempo tenemos que $\dot{x}=f(x, u(x))$. Para el caso en que el sistema es lineal en el estado y en el control, e invariante en el tiempo, la anterior ecuación se escribe como $x=A x+B u$, con $A$ y $B$ matrices constantes de dimensiones compatibles. Si se tiene realimentación lineal de estado $u(x)=K x$, donde $K$ es un vector fila de constantes que se calculan en función del comportamiento deseado, este sistema queda entonces expresado como $\dot{x}=(A-B K) x$. Puesto que en el sistema lineal autónomo $\dot{x}=P_{x}$ el comportamiento dinámico queda completamente determinado por los vectores y los valores propios de la matriz $P$, es evidente que con la realimentación de estado se consigue modificar el comportamiento de la planta.

Una realimentación con conjuntos de valores diferentes dependiendo de regiones previamente establecidas en el espacio de estado, conduce a dinámicas diferentes según la región en la que se encuentre el estado del sistema. Entonces se dice que el sistema es de estructura variable. Como caso particular considérese una función de control de realimentación de estado pero definidas de manera diferente según que el estado esté a uno u otro lado de una superficie $s(x)=0$ de dimensión $n-1$. Se tiene entonces,

$u(x)=\left(\begin{array}{lll}K^{+} x & \text { si } & s(x)>0 \\ \mathcal{K}^{*} x & \text { si } & s(x)<0\end{array}\right.$

donde los supraíndices sobre el vector $K$ distinguen los vectores de ganancia en las dos regiones separadas por la superficie. 
Un ejemplo simple de la situación descrita se obtiene con $A=\left[\begin{array}{ccc}0 & ; \\ a_{21} & 0\end{array}, \quad B=\left[\begin{array}{l}0 \\ 1\end{array}\right] \quad y\right.$ $u(x)=w\left(x_{1}\right)=t^{t} x_{1}$ según el estado esté encima - abajo de una superficie, lo que da dos estructuras centros $\dot{x}=A^{+} x$ y $\dot{x}=A \times$ con $A^{2}=\left[\begin{array}{cc}0 & 1 \\ a_{2}^{ \pm} & 0\end{array}\right.$ si $a_{21}^{t}=u_{21}+k^{t}<0$. Más explicitamente se tiénen dos sistemas de ecuaciones de primer orden, $\dot{x}_{1}=x_{2}: \dot{x}_{2}=a_{21}^{ \pm} x_{1}$, que reducidos a dos ecuaciones de segundo orden $\tilde{x}_{1}-a_{2}^{t}, x_{1}=0$, representan de manera evidente dos osciladores armónicos con frecuencias $\omega_{1}=\sqrt{\left.\mid a_{21}^{*}\right]}$ y $\omega_{2}=\sqrt{\left|a_{21}\right|}$. Evidentemente las soluciones son del tipo $x_{1}(t)=A \sin \omega t, x_{2}(t)=A \omega \cos \omega t$ de modo que la trayectoria en el espacio de fase es la elipse $x_{1}^{2} / A^{2}+x_{2}^{2} / \omega^{2} A^{2}=1$, Sobre la figura (1) se muestran superpuestas tres trayectorias con semieje mayor vertical, $\omega^{2}>1, y$ dos con semieje mayor sobre el eje $x_{1}$, esto es, con $\omega^{2}<1$, de tal manera que unas y otras tienen punto de tangencia. En estos puntos ocurre la conmutación y el estado sigue la trayectoria indicada por las flechas, hasta llegar al origen. La pregunta fundamental es de que manera ocurre la disipación de energía necesaria para llevar el estado hasta el origen, si las dos estructuras son conservativas e no se considera de manera explicita en el modelo, ningún elemento que disipe energia

2- EL ejemplo elemental descrito es clásico en la literatura de sistemas de estructura variable para ilustrar la posibilidad de obtener estabilidad asintótica ${ }^{1}$ a partir de dos estructuras que no tienen esta propiedad. Podemos redefinirio como

$$
\dot{q}=-\omega^{2} q \text { com } \quad \omega^{2}=\left\{\begin{array}{lll}
\omega_{1}^{2} & \text { se } & q \dot{q}>0 \\
\omega_{2}{ }^{2} & \text { se } & q \dot{q}<0
\end{array}\right.
$$

donde $q$ es posición y $\omega_{1}{ }^{2}>\omega_{2}{ }^{2}$ son las dos frecuencias de oscilación. Es claro que la conmutación se efectúa cuando la trayectoria del estado en el espacio de estado cambia de cuadrante, esto es, en los instantes en que posición

1. Estabilidad asíntotica implica adicional a la estabilidad, que el estado tiende a cero cuano el tiempo tiende a infinito. Para definiciones precisas ver referencias (3) y (4). retrato de fase para dos frecuencias

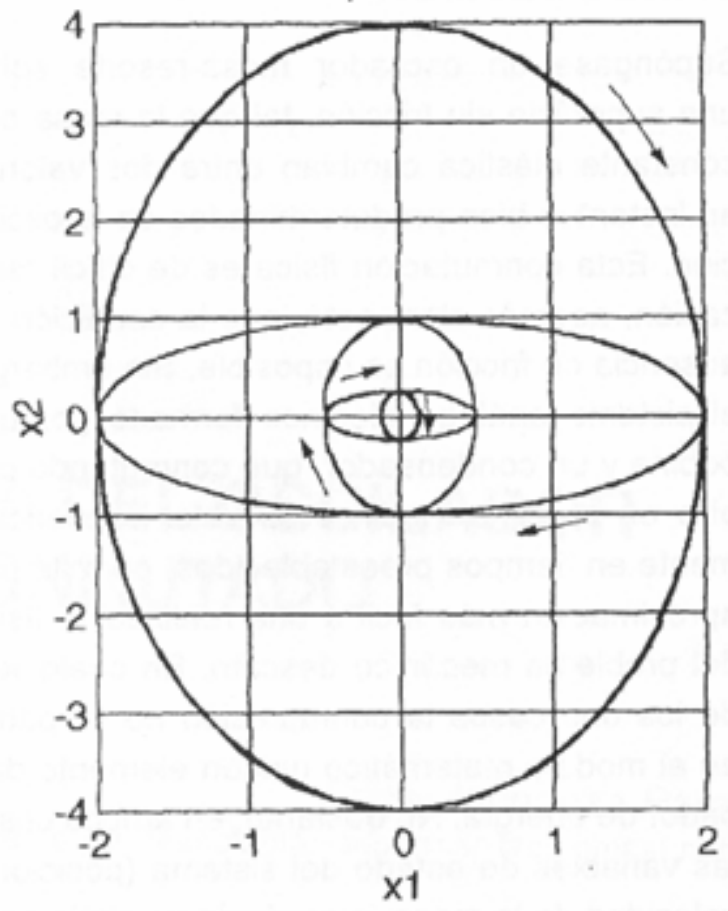

Figura 1: Estabilidad asintótica conmutando estructuras no estables asintóticamente

o la velocidad son cero.

3- La representación en las variables de estado posición q y momentum $p$ del oscilador armonico $\bar{q}+\frac{k}{m} q=0$, con función hamiltoniana equivalente a la energia mecánica total

$H(q, p)=\frac{1}{2} k q^{2}+\frac{1}{2 m} p^{2}$, es

$\left[\begin{array}{l}q \\ p\end{array}\right]=\left[\begin{array}{cc}0 & 1 / m \\ -k & 0\end{array}\right]\left[\begin{array}{l}q \\ p\end{array}\right]$

donde $m$ y $k$ son la masa y la constante elástica del resorte. En esta representación de estado la ecuación de cada trayectoria del retrato de fase en el plano $p q$ es la elipse

$\frac{q^{2}}{A^{2}}+\frac{p^{2}}{m^{2} A^{2} \omega^{2}}=1$

donde $A$ es la amplitud máxima de la oscilación y $\omega^{2}=k / m$.

4. El sistema fineal (1) es continuo por trechos y por to tanto puede ser analizado separadamente en cada trecho, observando después la continuidad de la solución. Como en cada trecho el sistema es conservativo, la única posibili- 
dad de explicar el comportamiento asintóticamente estable de la solución, es aceptar la pérdida de energia en los instantes en que ocurre la conmutación. Esta hecho es confirmado en el analisis que sigue (para las conmutaciones donde la velocidad es cero), a partir de la evaluación de la energía en los puntos extremos de cada trecho. En estos puntos el cálculo es directo debido a que con posición o velocidad nulas, la energia mecánica total es exclusivamente cinética o potencial.

\section{Variación instantánea de la energía del sistema.}

1. De la ecuación (4) es claro que la trayectoria del estado para el sistema de estructura variable formado por dos osciladores de frecuencias distintas conmutado según la ecuación (1), sigue (a partir de una condición inicial en el primer cuadrante) sucesivamente con cada conmutación, trechos de elipses con semieje mayor vertical y horizontal si

$$
\omega_{1}^{2}>\frac{1}{m^{2}}>\omega_{2}^{2} \cdot 1
$$

Como el estado no cambia instantáneamente, el punto de tangencia de las elipses es el valor del estado en el instante de la conmutación ${ }^{2}$. Así, el comportamiento asintóticamente estable del sistema como um todo, es ilustrado de manera evidente con un primer bosquejo sin necesidad de simulación. El tiempo para cada trecho es $\frac{2 \pi}{4 \omega_{i}}, i=1,2$. Para efectos de ilustración más exacta, la simulación de la figura (2) muestra el retrato de fase para $A=1.25, \omega_{1}=1.25$, $\omega_{2}=0.5 \omega_{1}, \quad m=2$ y condiciones iniciales $p(0)=A m \omega_{1}, q(0)=0 .{ }^{3}$ En la figura (2) se indican

1. Si se escogen como variables de estado, la posición y la velocidad, la representación de estado es

$$
\left[\begin{array}{l}
\dot{q} \\
\dot{q}
\end{array}\right]=\left[\begin{array}{cc}
0 & 1 \\
-\omega^{2} & 0
\end{array}\right]\left[\begin{array}{l}
q \\
\dot{q}
\end{array}\right]
$$

y la trayectoria sigue la ecuación. $(q / A)^{2}+(q / A \omega)^{2}=1$. Sin embargo en esta representación el Hamiltoniano $H(q, \dot{q})=\frac{2}{2}\left(q^{2}+\omega^{2} q^{-2}\right)$ no tiene interpretación de energía lo que es fundamental para la argumentación que sigue. las conmutaciones por números naturales, los trechos por letras y se muestran algunas cordenadas en el instante de cada conmutación. Estas son obtenidas facilmente mediante la expresión (4) si la condición inicial $x_{0}$ es escogida sobre los ejes de la elipse: por exemplo. para $x_{0}=\left(0, p=m \omega_{1} A\right)$ las coordenadas $\frac{2 \pi}{4 \omega_{1}}$ unidades de tiempo después, en el final del trecho antes de la conmutación, son $x\left(\frac{\pi}{2 \omega_{1}}\right)=\left(p / m \omega_{1}=A, 0\right)$. Si la condición inicial fuera $x_{0}=(q, 0)$ el estado en la siguiente conmutación será $x\left(\frac{\pi}{2 \omega_{2}}\right)=\left(0,-q m \omega_{2}\right)$. Nótese que en trechos sucesivos se alterna la frecuencia; el primer trecho, $a$, tiene frecuencia $\omega_{1}$.

2. El cálculo da energía a lo largo de la trayectoria es directo a partir del cálculo de las coordenas del estado en el comienzo y fin de cada trecho, y del carácter conservativo del sistema

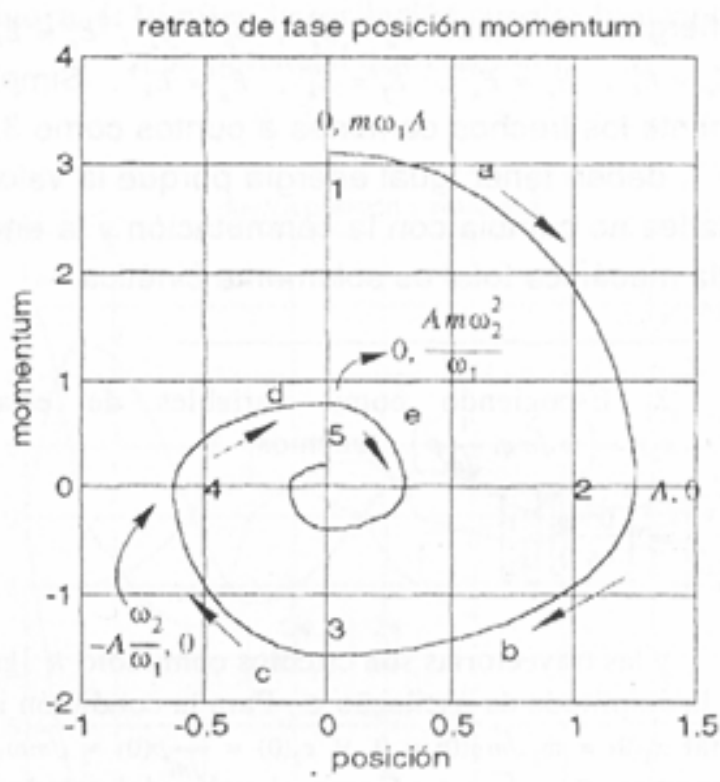

Figura 2: retrato de fase oscilador conmutado

en cada trecho. Como en el comienzo y fin de todos los trechos una de las coordenadas es cero, la energía puede calcularse a partir de la energía potencial $E_{p_{1}}=\frac{1}{2} k q^{2}=\frac{1}{2} m \omega^{2} q$ de la energia cinética $E_{k}={ }_{2} m q^{2}$. Por ejemplo, en el trecho $a$, la energia mecánica total es $E_{a}=E_{k}^{i+}=\frac{1}{2} m\left(A \omega_{i}\right)^{2} \circ E_{a}=E_{p}^{2-}=\frac{1}{2}^{m} \omega_{1}^{2} A$, donde el supraindice junto al numero de la conmutación indica si el instante considerado es anterior o posterior a la conmutación. 
Con la anterior notación, y calculando la energía en cada trecho siempre a partir de la energia potencial $E_{p}=\frac{1}{2} m \omega^{2} q$, tenemos que con $E_{a}=E_{p}^{2 *}, E_{b}=E_{p}^{2+}, E_{c}=E_{p}^{4}, E_{d}=E_{p}^{4 *}, E_{e}=E_{p}^{6 .}$. $E_{f}=E_{p}^{t *}$. etc., obtenemos fácilmente

$\frac{E_{a}}{E_{b}}=\frac{E_{c}}{E_{d}}=\frac{E_{e}}{E_{f}}=\ldots . .=\frac{\omega_{1}^{2}}{\omega_{2}^{2}}$

porque la coordenada de la posición en los trechos que se relacionan en la ecuación (6), es la misma, y la frecuencia se modifica. Por otra parte,

$E_{b}=E_{c} ; E_{d}=E_{;} ; E_{f}=E_{g} \ldots \ldots$

ya que la diferencia de coordenadas "se compensa" con la diferencia de frecuencias. Las relaciones indicadas en (7) también se visualizan inmediatamente con los cálculos a partir de energías cinéticas $E_{a}=E_{k}^{1+}, E_{b}=E_{k}^{3+}, E_{c}=E_{k}^{3+}$, $E_{d}=E_{k}^{5+}, E_{\mathrm{r}}=E_{k}^{5+}, E_{f}=E_{k}^{7 .}, E_{g}=E_{k}^{7+}$. Simplemente los trechos comunes a puntos como 3,5 ○ 7, deben tener igual energia porque la velocidades no cambia con la conmutación y la energía mecánica total es solamente cinética.

2. Escogiendo como variables de estado $\left(x_{1}, x_{2}\right)=\left(\omega \sqrt{m} q, \frac{1}{\sqrt{m}} p\right)$, tenemos

$\left[\begin{array}{l}x_{1} \\ x_{2}\end{array}\right]=\left[\begin{array}{cc}0 & \omega \\ -\omega & 0\end{array}\right]\left[\begin{array}{l}x_{1} \\ x_{2}\end{array}\right]$

y las trayectorias son círculos com radio $R$ igual a la amplitude da oscilação $C$. Para la condición inicial $x_{1}(0)=\omega_{1} \sqrt{m} q(0)=0$ y $x_{2}(0)=\frac{1}{\sqrt{m}} p(0)=\sqrt{m} \omega_{1} A$. tenemos $c=\sqrt{m} \omega_{1} A$. Como el való del estado no cambia en el instante de la conmutación, tendríamos siempre trayectorias sobre el mismo círculo y aparentemente la conclusión sobre estabilidad asintótica no sería posible. La solución a este dilema es dada por la relación entre el área $S$ del circulo y la energía en esta representación de estado. El área del círculo es $S=\pi R^{2}=\pi m \omega_{1}^{2} A^{2}$ y la energía mecánica total calculada a partir de la potencial en el instante de amplitud máxima es $E=\frac{1}{2} k A^{2}=\frac{1}{2} m \omega_{1}^{2} A^{2}$. de modo que $E=2 \pi S$. Con el valor de $S$ anotado, la relación de energías entre el primero y el segundo trecho es

$\frac{E_{1}}{E_{2}}=\frac{\omega_{1}^{2}}{\omega_{2}^{2}}$,

lo que indica la perdida de energía
3. La relación entre energia y el área de la elipse definida por la trayectoria cerrada en el espacio de estado del sistema (3) también es

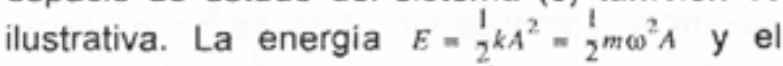
área de la elipse es $S=\pi m \omega A^{2}$ de modo que se obtiene $E=S \omega / 2 \pi$ y entonces,

$\frac{E_{a}}{E_{c}}=\frac{S_{c} \omega_{1}}{S_{c} \omega_{1}}=\frac{\pi m \omega_{1} A^{2}}{\pi m \omega_{2} A A\left(\omega_{2} / \omega_{1}\right)}=1$ mientras que

$\frac{E_{a}}{E_{b}}=\frac{S_{a} \omega_{1}}{S_{b} \omega_{2}}=\frac{\pi m \omega_{1} A^{2} \omega_{1}}{\pi m \omega_{2} A^{2} \omega_{2}}=\frac{\omega_{1}^{2}}{\omega_{2}^{2}}$.

De las relaciones (6) e (7) se observa que la disminuición de la energía sucede solo en las conmutaciones donde la velocidad es cero, o sea cuando la masa está en la posición de amplitud máxima y mínima.

4. La suma de las infinitas perdidas de energía debe ser igual a energía inicial. En efecto, considerando que las relaciones entre los valores de energla después de cada conmutación i donde ocurre pérdida de energía son

$\frac{E_{i+1}}{E_{i}}=r^{2}=\left(\omega_{2} / \omega_{1}\right)^{2} \quad i=0,1,2 \ldots \infty$.

se forma la serie

$\sum \Delta E=E_{0}\left(1-r^{2}\right)\left(1+r^{2}+r^{4}+r^{6}+\ldots.\right)$.

que converge a $E_{0}$, el valor de la energia inicial.

En la figura (3) se ilustra la conservación de la energía en cada trecho y las sucesivas diminuciones en los instantes donde la energía cinética es cero, para los valores de la simulación de la figura (2).

\section{Interpretaciones físicas: la naturaleza de la fuerza.}

1. Es ilustrativo observar el movimiento sobre la función de energia potencial a la manera clásica como es efectuada para determinar los limites del movimiento en sistemas conservativos. En nuestro caso, sobre las dos funciones de potencial definidas por las conmutaciones.

3. El primer trecho transcurre en una elipse "parada". Obsérvese que la escala horizontal y verticalen la figura, son diferentes. 


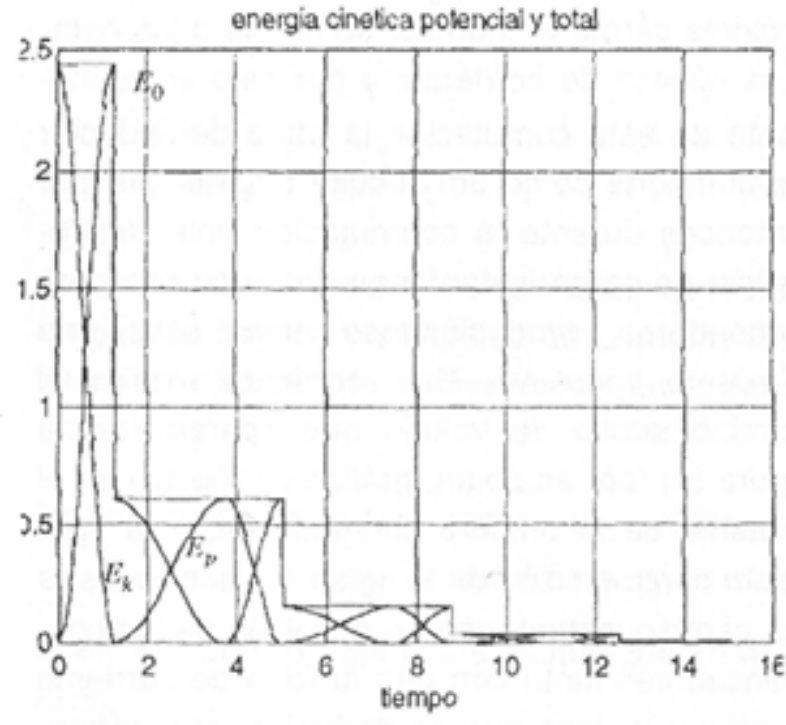

Figura 3: Variación de la energía mecánica total

Se trata de construir a partir de una energia inicial $E_{0}$, los limites de la oscilación de una particula entre dos funciones de potencial que conmutan. Las dos funciones de potencial son $U_{1}(q)=\frac{1}{2} m \omega_{1}^{2} q$ y $U_{1}(q)=\frac{1}{2} m \omega_{2}^{2} q$. La posible trayectoria se muestra en la figura (4), indicando con letras los mismos trechos mostrados en la figura (2). Obsérvese la variación finita de la fuerza elástica (derivada da función potencial) en los puntos de amplitud máxima de elongación del resorte y la reducción finita de energía en esos mismos puntos.

Finalmente, la figura (5) muestra la posición $q$, la velocidad q y la fuerza elástica $f=-k q=-m \omega^{2} q$. Obsérvese la discontinuidad de la fuerza en los instantes de la conmutación y el salto solamente en las conmutaciones donde la velocidad es máxima y la posición es cero ${ }^{1}$.

Sin embargo, la fuerza en los puntos de discontinuidad no es impulsiva pues no hay variación instantánea de la cantidad de movimiento. La variación instantánea de la fuerza es de dificil interpretación física en el sistema masa resorte analizado porque estará asociada a una función

1. La forma y valores de la figura (5) cs completamente previsible sin simulación. los saltos aparecem porque en los instantes de la conmutación con posición máxima, la fucrza varía instantaneamente debido al cambio de freqücncia.

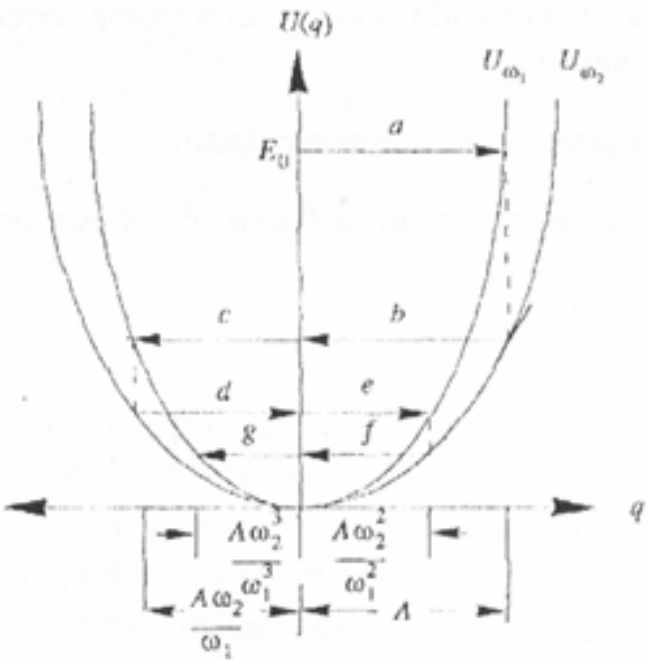

Figura 4: Limites da oscilación con dos funciones de potencial que comutan

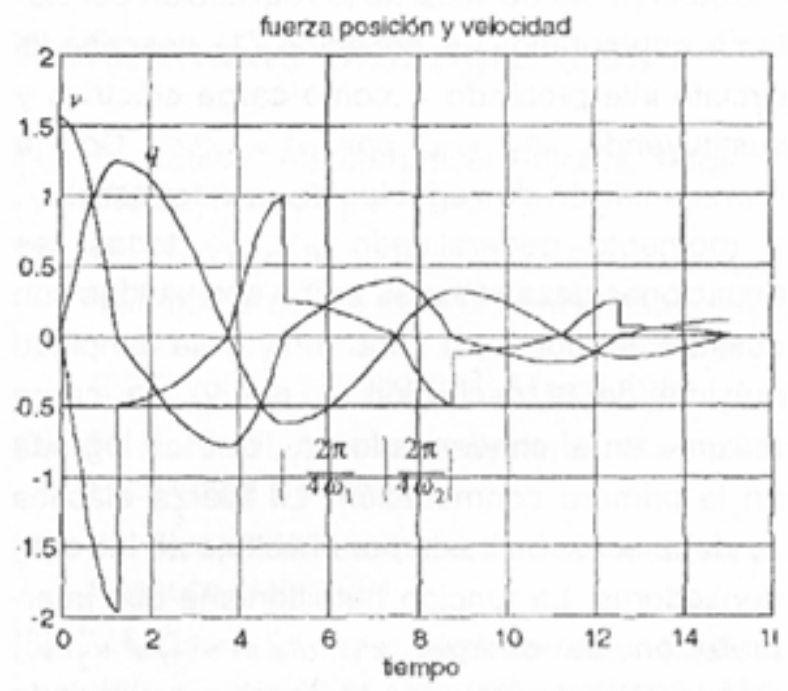

Figura 5: fuerıa elástica, posición y velocidad

impulsiva de magnitud masa multiplicada por la derivada de la aceleración, de manera que $\int \delta_{m a}=F^{2}$ Una analogía eléctrica para la fuêrza es el voltaje. Como la variación instantánea del voltaje en un condensador es de inter-

2. 2. Si la fuerza fuera impulsiva tendríamos $\int \delta_{F}=i=m\left(v_{2}-v_{1}\right)$. El subíndice en la función impulso indica su unidad física. 
pretación directa, y está asociada a una corriente impulsiva, se estudia a continuación el oscilador conmutado análogo al sistema mecánico analizado.

\section{El oscilador eléctrico conmutado.}

El circuito eléctrico de la figura (6) ofrece una

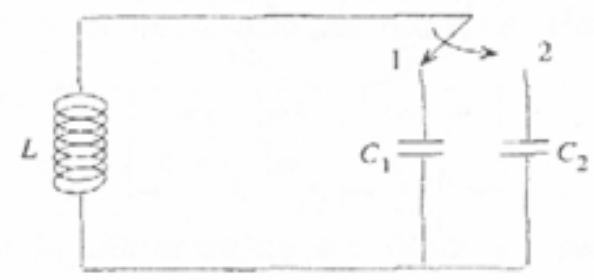

Figura 6: Oscilador armónico conmutado eléctrico

interpretación física más completa y razonable desde el ponto de vista de la realización del sistema conmutado. La ecuación (1) describe el circuito interpretando $q$ como carga eléctrica $\mathrm{y}$ sustituyendo $\omega^{2}=k / m$ por $\omega^{2}=1 / L C$. Con la representación de variables de estado, carga $q$, y momento generalizado $p=L \dot{q}$, todas las ecuaciones desarrolladas arriba son válidas con sustituir $m$ por $L, k$ por $1 / C$ y la amplitud máxima de la oscilación $A$ por $Q$, la carga máxima en el condensador $C_{1}$, que es lograda en la primera conmutación. La fuerza elástica $k q$ debe ser substituida por el voltaje en los condensadores. La función hamiltoniana con interpretación de energía es $H(q, p)=\frac{1}{2 C} q^{2}+\frac{1}{2} L \cdot q^{2}$, siendo el primer término la energía acumulada en el campo eléctrico del condensador activo, y el segundo término la energía acumulada en el campo magnético del inductor. Todas las figuras también son válidas con las sustituciones anotas. Las figuras (2) a (5) se interpretan de la siguiente manera: el primer trecho con el interruptor en la posición $1 \mathrm{y}$ el condensador $C_{1}$ conectado, se inicia con toda la energía en el inductor (coordenadas: $0, L \omega, Q$ ) y finaliza con toda la energía en el condensador $C_{1}$, que tiene en el instante de la primera conmutación la máxima carga. El inductor se opone a los cambios súbitos de corriente, y por esto en el instante de esta comutación la rama del inductor se comporta como um circuito abierto. Sucede entonces durante la conmutación una transferencia de carga instantánea entre los dos condensadores, produciéndose entre ellos una corriente impulsiva. Esta corriente explica el cambio súbito de voltaje que aparece en la figura (5) (por analogia, gráfico de fuerza) en el instante de la primera conmutación, y la aparente contradicción de la figura (5) que muestra un cambio instantáneo en el voltaje de los condensadores junto con una función de corriente continua (si bien que no derivable) que permanece en cero durante la conmutación. La explicación para esta contradicción aparente es que en la figura (5) se muestra la corriente que circula por el inductor y no el impulso de corriente entre los dos condensadores, que ocasiona la variación instantánea finita de voltaje. ${ }^{1}$ La transferencia de cargas instantánea entre los dos condensadores implica una disminuición de energía como se verá en el siguiente item.

En la segunda conmutación el cambio de condensadores sucede cuando estan descargados de modo que entre ellos no hay ninguna transferencia de carga. El único efecto es el cambio de la frecuencia de oscilación.

3. Transferencia de carga instantánea entre dos condensadores.

Considérese la conexión de dos condensadores $C_{1}$ e $C_{2}$ con voltajes diferentes $v_{1}^{*}=q_{1} / C_{1}$ y $V_{2}^{*}=q_{2} \cdot / C_{2}$ antes de la conexión. En el instante posterior a la conmutación los voltajes deben ser iguales a $V^{+}=\left(q_{1}{ }^{+}+q_{2}\right) /\left(C_{1}+C_{2}\right) \cdot{ }^{2}$ Considerando la conservación de la carga $q_{1^{*}}+q_{2}=q_{1}+q_{2}$ tenemos que las cargas en los condensadores después de la conmutación son

1. El condensador "se opone a los cambios súbitos da voltaje", a menos que la corrente sea um impulso, que es precisamente lo que sucede entre los condensadores en el instante de la transferência de las cargas

$$
\text { 2. } q_{1}^{+}+q_{2}^{+}=C_{1} v_{1}^{*}+C_{2} v_{2}^{+}=\left(C_{1}+C_{2}\right) v^{+} \text {. }
$$


$q_{1^{\prime}}=V^{+} C_{1}=\left(q_{1}+q_{2}\right) \frac{C_{1}}{C_{1}+C_{2}} \mathrm{y}$

$q_{2}^{+}=v^{+} C_{2}=\left(q_{1}^{*}+q_{2}^{*}\right) \frac{C_{2}}{C_{1}+C_{2}}$.

Asi, tenemos una transferencia instantánea de carga que implica un impulso de corriente con intensidad igual a la diferencia de las cargas, esto es, $\int \delta_{i}=\Delta q$.

La energia antes de cerrar el circuito es $E^{*}=q_{1}^{2} / 2 C_{1}+q_{2}^{2} / 2 C_{2}$ e inmediatamente después es, considerando los resultados (8.a) y (8.b),

$$
E^{*}=q_{1}^{2}, / 2 C_{1}+q_{2}^{2}, / 2 C_{2}=\left(q_{1}-q_{2}\right)^{2} / 2\left(C_{1}+C_{2}\right),
$$

y por lo tanto la perdida de energía en la conmutación es

$$
\Delta E=\frac{\left(q_{1} C_{2}-q_{2} C_{1}\right)^{2}}{2\left(C_{1}+C_{2}\right) C_{1} C_{2}} .
$$

Si los dos condensadores son iguales, la ecuación (9) se reduce a $\Delta E=\left(q_{1},-q_{2},\right) / 4 C$ que se hace cero si antes de la conmutación las dos cargas (o los dos voltajes) de los condensadores son iguales. En ese caso, en el instante de cerrar el circuito no hay corriente, lo que sugiere claramente que la pérdida de energía está asociada al impulso de corriente de la transferencia instantánea de cargas.

Esto es más plausible estudiando el proceso de transferencia de cargas entre dos condensadores conectados a través de una resistencia y calculando la energía disipada en la resistencia $R$ durante el tiempo del transitorio. En el limite cuando la resistencia tiende a cero tendríamos nuestro caso de estudio. Considérese el circuito descrito con cargas iniciales $q_{1} \neq 0$ e $q_{2}=0$.

En este caso la corriente es $i(t)=\frac{q_{1} / C_{1}}{R} e^{-t / R C_{c e}}$. donde $C_{e q}=C_{1} C_{2} /\left(C_{1}+C_{2}\right)$ es la capacitancia equivalente de los dos condensadores en serie vistos desde la resistericia. La energía disipada por la resistencia $W=\int_{0} i^{2}(t) R d t$ es igual a
$W=\frac{1}{2} q_{1}^{2} \cdot \frac{C_{2}}{C_{1}\left(C_{1}+C_{2}\right)}$

que es la energía inicialmente acumulada en el condensador. La integral que da como resultado (10) no depende de la resistencia y coincide con $(9)$ en el caso $q_{2}=0$.

Obsérvese que la potencia $p(t)=\frac{\left(q_{1} / C_{1}\right)^{2}}{R} e^{-2 r / R C_{*}}$

es una función impulsiva cuando $R \rightarrow \infty$, esto es, una amplitud infinita con una integral finita. Esto responde nuestra pregunta básica sobre la perdida de energía en um modelo donde no existen elementos disipativos. Asi, coloquialmente podemos explicar la perdida de energía como el resultado de un proceso límite de disipación en tiempo cero, de una potencia indeterminada formada por una corriente que tiende a infinito a través de una resistencia que tiende a cero.

\section{Referencias Bibliográficas}

[1] E. Butkov. Mathematical Physics. Addison Wesley. New York. 1973.

[2] R. DeCarlo, S. Zak.,S, G. P. Matthews. Variable sructure control of nonlinear multivariable systems: a tutorial. Proceedings IEEE.vol 76, (3):212-231. March 1988.

[3] H. K. Khalil. Nonlinear Systems. Macmillan Publishing Co. 1992.

[4] J.J Slotine, W. Li. Applied nonlinear control. Prentice.Hall. 1991.

[5] V.I. Utkin. Sliding modes and their application in variable structure systems. Mir. 1978.

[6] V.I. Utkin. Variable structure systems with sliding modes. IEEE Trans. Automat. Contr., AC-22, (2):.212-222, April 1977. 